Article

\title{
Symmetry of Galactic Structure
}

\author{
Richard Oldani
}

retired

Correspondence: oldani@juno.com

\begin{abstract}
A fully relativistic description of an atomic clock is achieved by applying the equivalence principle directly to the space-time coordinates of electron transitions. The resulting differential equation of motion is then reformulated by means of Hamilton's principle to obtain an equivalent integral equation. Generalized coordinates describe the electron in a configuration space consisting of three coordinates that define the origin, or nucleus, and three coordinates that define the manifold on which the electron is constrained to move (the electron shells). Following Dirac the particle model is transformed to a field model by describing energy quantization as the four-dimensional localization of radial and transverse electromagnetic fields. It can then be compared to the emission energy of galaxies in terms of the radial baryonic fields and transverse gravitomagnetic fields that emanate from black holes. The symmetry exhibited by these diverse forms of energy demonstrates that the properties of energy, when described four-dimensionally with time, are independent of the material system which supports them.
\end{abstract}

Keywords: time; gravitomagnetics; mass-energy equivalence; quantum mechanics; relativity theory; symmetry; inertial and non-inertial coordinates; space-time linearity

\section{Introduction}

We do not have a theory of quantum gravity that successfully describes both quantum mechanics, governing the microscopic properties of matter; and relativity theory, its large scale behavior. It is hypothesized here that the greatest impediment to integrating these two foundational frameworks of theoretical physics is the question of time. Time is registered by clocks, devices that read out a one-dimensional sequence of increasing numbers. Currently there is no way to distinguish between clocks, a mechanism, and time, a concept. They are treated operationally as one and the same thing. On the one hand clock mechanisms are quantum oscillators consisting of electrons that transition between orbitals while registering time as a series of ticks. On the other hand, time is relativistic in nature and can vary according to a clock's motion and gravitational potential. The clocks in GPS systems are corrected for velocity and gravitational potential, but without knowing from a quantum mechanical perspective how it happens. In other words, how is it possible for clocks to register ticks absolutely while time is perceived relativistically? Moreover, corrections made to the orbiting clocks due to velocity and gravitational potential are applied simultaneously. If clocks are conceived of as operationally singular mechanisms that indicate time with a string of ticks caused by a transitioning electron, then it is difficult to comprehend how the electron, transitioning within the atom in a regular way, is able to process inputs from two physically distinct external sources, velocity and gravitational potential.

\section{Inertial and non-inertial frames}

Time is not an observable in quantum mechanics. Observables are measured by bringing a measuring device into contact with the physical system and different values of the observable are obtained depending on the state of the system. In quantum mechanics time measurements are performed without making contact with the observed physical system, a clock. So the time "measured" by clocks is not a quantum mechanical observable. 
It is just a numerical parameter, one of four coordinates, the same as in classical physics, and clocks are treated as simple measuring devices of absolute time. If time could actually be treated the same as spatial parameters, then a simple transformation of clock coordinates would be sufficient to make the quantum and relativity theories compatible. However, relativistic time varies with respect to velocity and gravitational potential, and this contrasts sharply with quantum mechanical clocks which measure time in absolute increments. To understand why there is a fundamental difference between quantum mechanical time and relativistic time it is necessary to look at the origins of quantum theory.

Soon after the Bohr model of the atom was proposed in 1913 the assumption was made that the effects of gravitational fields on an atom during the emission and absorption of radiation was so small that they could be neglected. At the time there were very few objections and no hard evidence to the contrary. The uncertainty principle was introduced upon further development of the quantum mechanical formalism providing "proof" and it contributed to the popular belief that a nonrelativistic formulation of quantum mechanics for atomic structure is both sufficient and complete. This caused the dynamic, classically inspired geometry of relativity theory to be set aside in favor of Hilbert space, an abstract complex linear vector space that is rigid. Because quantum theory is limited to applications in inertial systems there was no immediate need for it to be extended to non-inertial geometries. However, in recent years new and totally unexpected experimental techniques have been developed that can link 10,000 atoms in a lattice that functions as a single quantum oscillator and atomic clock [1].This allows error due to thermal radiation to be essentially eliminated from properties of atomic structure and extremely precise measurements of time to be performed. Quantum oscillators of this type are able to detect differences in the earth's gravitational potential as small as one centimeter which suggests that time coordinates are to be treated the same as spatial coordinates with a different value at each point in space [2]. It means that even the tiniest of gravitational potentials cannot be ignored; and that in fact, all physical laws should be formulated in non-inertial frames.

Nonrelativistic quantum mechanics is formulated in inertial frames; that is, frames in uniform relative motion and in the absence of gravitational potentials. To be applied in non-inertial frames it must first be formulated relativistically. Dirac noted that possibility in his paper on quantum electrodynamics [3], "The theory is non-relativistic only on account of the time being counted throughout as a c-number [classically], instead of being treated symmetrically with the space coordinates." In other words, a relativistic theory should not treat time continuously, but as a variable with both discrete and continuous properties the same as the space coordinates. Once a fully relativistic theory has been formulated such that time is treated symmetrically with the space coordinates it will hold in all frames, also accelerated frames. We can then use it to apply the equivalence principle to electron transitions in accelerated frames. In other words, we can make quantum mechanical clocks consistent with the relativistic time we perceive by transforming from a nonrelativistic to a relativistic theory.

\section{Gravitational perturbation}

\subsection{Standard model}

According to the standard model discrete exchanges of gravitational field energy occur at the quantum level and they may be studied by the detection of gravitons; however, an experimental procedure that could be performed in practice has never been developed. Because of their low cross section during interactions with matter it has been questioned whether the detection of gravitons is even possible [4]. An alternate method is to use the field model in continuum space as advocated by Einstein and study the behavior of physical systems at the quantum level by perturbing them with a barely detectable gravitational field. A light beam that alters direction in propagation space by close proximity to a massive body is one example. A second example is the perturbation that occurs when a slight difference in gravitational potential causes an atomic clock to change its rate. In 
order to study slight changes in clock rate caused by gravitational perturbation an interpretation of quantum mechanics is required that includes time as a variable.

\subsection{The flat background space}

In general relativity theory space-time geometry curves in response to the influence of matter immersed in it. If space is significantly curved it would indicate that the background space-time is non-linear and all measurements would be distorted. However, deviations from linearity by light rays are not measured in curved spacetime. The curvature at any point is determined by measuring a tiny angle of deviation with respect to an imaginary orthogonal set of measuring rods. It means that curved space-time geometry is at times falsely claimed to act as a "background" for quantum phenomena in theories of quantum gravity because it is itself mapped with respect to Euclidean space. An imaginary framework of linearly oriented, orthogonally configured measuring rods is used in practice to determine how a gravitational field causes light rays to bend but it may be discounted afterwards as a simple calculational device that is non-physical. If the linearity of the underlying space deviated so extremely that it affected matter at the quantum level it is questionable whether we would be able to use it in practice to perform measurements at the macroscopic level.

Red shifts are used to determine the distance of luminous objects, their relative age, and the rotational velocity of galaxies. The time delay of a laser beam is used to determine our distance from the moon. If even an infinitesimal non-linearity were present as, for example, tiny differences in the homogeneity of space, the speed of light, or the properties of intervening matter; the billions of years in transit time of starlight would amplify them making useless attempts to assemble and compare data obtained by different collection methods or with respect to distinct wavelengths. Although the linearity of electromagnetic radiation during propagation is used to perform astronomical measurements at accuracies far beyond our ability to quantify them, it is not used at all at the microscopic level. Methods of renormalization ignore linearity in their interpretation of the behavior of quantum systems as a sum over histories or path lengths, and the same is true for entanglement phenomena which dismiss linearity altogether for wave function collapse. Theories that ignore the linearity of space at the microscopic level are not consistent with macroscopically obtained astronomical observations and will be set aside to be interpreted at a later point.

\subsection{Gravitational perturbation of clocks}

It is not possible to use the absolute time of a single clock to compare measurements in non-inertial systems such as occur in distinct or variable gravitational fields. To compare physical systems with differing gravitational potentials we use two separate clocks, one as measuring device and another as system. Let a laboratory clock $T_{L}$ that is fixed in space serve as measuring device and let a second movable clock Ts be introduced as physical system. The system clock Ts may be subjected to different gravitational potentials and then compared with the laboratory clock $T_{L}$ to determine how the clock rate changes. Clocks placed in locations of higher gravitational potential speed up with respect to a stationary clock, while clocks in uniform relative motion slow down. Thus, the state of the system $\mathrm{T}_{\mathrm{s}}$ is determined by its velocity and its gravitational potential, and measurements of the time coordinate with respect to $\mathrm{T}_{\mathrm{L}}$ are performed without making physical contact with the system which is normal procedure for time measurements in both relativity theory and classical theory.

The same property of light that allows astronomers to measure the distance of objects at the edge of the universe (spatial linearity) and compare the period of oscillation of red shifts (temporal linearity) can now be used to extrapolate to the very small dimensions of an atomic clock to analyze space-time at the microscopic level. The linearity of the radiation allows the coordinate differences of oscillating electrons in atomic clocks to be easily measured with respect to a flat space-time. Consider a well-known test for relativity 
theory using cesium beam atomic clocks. The clocks are flown around the world first in an eastward and then in a westward direction, and later compared to a laboratory clock fixed on the earth's surface [5]. The experiment demonstrates a slowing of clocks $\Delta \mathrm{t}_{\mathrm{k}}$ that is greatest in the eastward direction of flight due to the earth's rotation and a speeding up of clocks $\Delta \mathrm{t}_{\mathrm{g}}$ due to a decreased gravitational acceleration at higher altitudes. They are relativistic corrections to the time of the system clock Ts in the airplane which cannot be described nonrelativistically because they are located in a noninertial system of coordinates. When $\Delta \mathrm{tk}_{\mathrm{k}}$ and $\Delta \mathrm{tg}_{\mathrm{g}}$ are summed at the end of the flight they will equal the time on the atomic clock $\mathrm{T}_{\mathrm{L}}$ in the laboratory.

$$
\mathrm{T}_{\mathrm{L}}=\mathrm{Ts}_{\mathrm{s}}-\Delta \mathrm{tg}_{\mathrm{g}}+\Delta \mathrm{tk}_{\mathrm{k}}
$$

Because the clock rate is locally determined the frequency of the cesium atoms, $v_{c s}=$ 9,192,631,770 Hz, does not change during the approximately one week duration, $\Delta \mathrm{T} \approx$ $6 \times 10^{5} \mathrm{sec}$, of the flights. However, during that time period Ts speeds up relative to TL an amount equal to approximately $2.7 \times 10^{-7}$ seconds due to the combined influence of clock velocity and gravitational potential. The corrections are due to the relative velocity and altitude of the airplane, so they occur simultaneously.

The linearity of time allows the classical interval $\Delta \mathrm{T}$, or time of flight of the airplane, to be subdivided into a series of identically constituted clock cycles of period $\tau$ that sum linearly.

$$
\Delta T=T_{m}+T_{m+1}+. .+T_{n-1}+T_{n}
$$

The clock periods $\tau$ correspond to single ticks of the clock, complete cycles of a transitioning electron between excited and ground states. Neglecting kinematical corrections, each of them includes an infinitesimal correction with respect to the laboratory clock that when summed gives the correction factor for gravitational potential $\Delta \operatorname{tg}_{g}$ in 1). The maximum correction for each clock cycle is calculated by dividing the total correction by the approximate number of oscillations.

$$
\frac{\Delta t_{g}}{(\Delta T)\left(v_{C s}\right)} \leq 2.7 \times 10^{-23} \mathrm{sec}
$$

This corresponds to a path length of $\mathrm{ct} \leq 8.1 \times 10^{-15} \mathrm{~m}$ a distance that is much smaller than limits set by the uncertainty principle due to the wavelength, $\lambda=3.26 \times 10^{-2} \mathrm{~m}$, which sets a lower limit on the electron's distance from the nucleus. We interpret it instead as the maximum thickness of the electron shell. Although we cannot measure the coordinates of an electron that accurately nevertheless that level of precision is necessary in order to account for the linearity of time at the microscopic level. Thus, relativity theory demands a precision in the structure of the cesium atom that is at least nine orders of magnitude greater than the uncertainty principle. Because the linearity of space-time requires a greater accuracy for atomic structure than quantum mechanics allows the legitimacy of theories that describe space-time as having a granular structure is questioned.

\subsection{Improved experiments}

An improved measurement was made of clock rate differences between optical atomic clocks separated by a difference in height of $0.33 \mathrm{~m}$ [6]. After 40,000 seconds of data the authors found that due to the difference in gravitational potential the clocks exhibited a "fractional frequency shift" of $4.1 \times 10^{-17}$ cycles/second. The frequency shift is caused by the difference in gravitational potential between atomic clocks. This corresponds to a fractional shift in wavelength $\Delta \lambda$.

$$
\Delta \lambda=\frac{\Delta f}{f} \times \lambda=\left(4.1 \times 10^{-17}\right)\left(2.6 \times 10^{-2} \mathrm{~m}\right)=1.06 \times 10^{-18} \mathrm{~m}
$$

The fractional shift in wavelength represents an improved measurement of the accuracy that atomic coordinates must have in order to reflect a difference in height of $.33 \mathrm{~m}$. It is more than 29 orders of magnitude smaller than the uncertainty relation allows, since minimum uncertainty in position is given by the wavelength of the light. 
The most recent and also most accurate clock experiments use a single crystal and ultraviolet light to differentiate between the gravitational potential of the crystal's upper and lower surfaces, a distance of one millimeter [7]. Using the fractional frequency instability given for that experiment, $4.4 \times 10^{-18}$, we are able to determine the maximum thickness of the electron shell due to fractional wavelength.

$$
\Delta \Lambda=\frac{\Delta f}{f} \lambda=\left(4.4 \times 10^{-18}\right) \times\left(6.98 \times 10^{-7} \mathrm{~m}\right)=5.3 \times 10^{-25} \mathrm{~m}
$$

Based on this experiment and proposed improvements to achieve even greater clock accuracy, space-time is hypothesized to be linear and homogeneous to infinitesimally small distances, and as a result continuously differentiable. The results of calculations for fractional wavelength provide compelling evidence to support a continuous rather than granular structure for space-time, and also support for the linearity of space-time, important founding elements for theories of quantum gravity.

\section{Lagrangian quantum mechanics}

\subsection{Electron decay}

Experiments cited in the previous section show that space-time geometry is linear at the microscopic level. Therefore the general theory of relativity is unnecessary for interpreting the influence of gravity on a quantum oscillator at the local level. Frequency shifts have been measured in the laboratory and they show that the laws of special relativity govern the propagation of light in free-falling frames [8]. Since no observable distinction can be made between accelerated motion and motion under the influence of a gravitational force the equivalence principle can be applied locally to the coordinates of a single quantum oscillator residing in Minkowski space. From equation 1) and the experimentally assured linearity of light we conclude that the electron of the quantum oscillator Ts oscillates at frequency $v c_{s}$ with relativistic correction to each cycle $\Delta t(\dot{x})$ due to angular velocity relative to the center of the earth and correction to each cycle due to an acceleration of coordinates $\Delta t(\ddot{x})$ between ticks of the clock.

A spatially determined correction of electron coordinates due to the equivalence principle is not possible inside an atom because atomic structure is an invariant. However, according to special relativity theory the space and time coordinates are interchangeable [7]. To obtain the necessary manifestly covariant equation in four dimensions we apply the equivalence principle to electron coordinates with time as a dependent variable and spatial coordinates as parameters. The correction factors for the classical interval $\Delta \mathrm{T}$ in 1 ) can be extrapolated to the level of the quantum oscillator to obtain a differential equation for the variation of time with respect to space for each cycle.

$$
f[t(x)]=\Delta t(\dot{x})-\Delta t(\dot{x})
$$

where $\Delta t(\dot{x})$ represents a kinematical time dilation and $\Delta t(\ddot{x})$ represents a speeding up of time due to acceleration.

Hamilton's principle states that the differential equations of motion for any physical system can be reformulated as an equivalent integral equation. To obtain an integral equation describing the electron's path from 3) we introduce the action integral $S[q(t)]$, a functional, which takes a function of the velocities (the summed velocity of earth and airplane) and coordinates (gravitational potential) as input and returns a scalar.

$$
S[q(t)]=\int_{0}^{t} L(\dot{q}(t), q(t), t) d t=h
$$

We interpret 4 ) in flat space-time as the evolution $\mathbf{q}(t)$ of a quantum system between the times of a complete cycle 0 and $t$, where $\mathbf{q}$ represents the generalized coordinates. The generalized coordinates describe the electron's location in a configuration space consisting of three coordinates that define the origin, or nucleus, and three coordinates that define the manifold on which the electron is constrained to move in three-dimensional space (the electron shells). In other words, the quantum oscillator is described in configuration 
space as having six parameters, three for the nucleus and three for the electron shells, a total of six degrees of freedom. Solving for the action we obtain an equation describing single cycles, which are the individual ticks of an ideal clock.

\section{$\mathrm{E} \tau=\mathrm{h}$}

By treating time symmetrically with the space coordinates a relativistic theory describing atomic structure is obtained that accurately describes the behavior of a quantum oscillator. The endpoint of an oscillation is conceived of as an event on an electron shell which designates a tick of the clock. The action $S[x(t)]$ describes a complete cycle of the quantum oscillator by taking into account a gravitational potential $\Delta t(\ddot{x})$ that accelerates the electron and a kinematic component $\Delta t(\dot{x})$ due to its continuous motion. Because they appear in the same equation two sets of coordinates are needed. Thus, atomic structure requires two complete four-dimensional space-times as a direct result of the equivalence principle; discrete coordinates that denote electron shells and continuous coordinates that describe an electron's continuous motion during transition.

Quantization is described as a four-dimensional transformation of fields between electron shells, the volume enclosed by the manifold. By treating time symmetrically with the space coordinates a relativistic theory of atomic structure is obtained that is superior to nonrelativistic theory because it accurately reflects influences of velocity and acceleration on the time coordinate. It is a classically derived model that describes space-time discretely in the Minkowski space of the atom, while the intervening space-time in which atoms reside and move is continuous and curved. Despite vast amounts of experimental evidence demonstrating that energy quanta are discrete it does not follow logically that space-time is also discrete as it is portrayed in string theory and loop quantum gravity. They are not equivalent concepts. Energy resides in space-time, is contained by it, and is subordinate to it. This is expressed formally by 4) which describes quantization as a localization of energy within the space-time manifold of an atom's configuration space, an electron shell. It distinguishes the Minkowski space of the atom from the curved space of gravitational fields that atoms reside in.

\subsection{Relativistic emission of energy}

It is not possible to make a direct comparison of gravitational fields to electromagnetic fields. Not only are they of vastly different strengths, but gravitational potentials do not share differences in polarity that are characteristic of electric charge. Despite glaring differences in their gross outward physical appearance, there are similarities that exist on a more subtle level. The kinetic flow of mass whether linear or rotational creates a transversely directed gravitational field that is analogous to the transverse magnetic field caused by current flow. The induced gravitomagnetic field, or force is directed perpendicular to the mass flow and has been used to explain properties of relativistic jets emanating from the cores of quasars and other active galactic nuclei [10]. It is postulated here that due to mass-energy equivalence an analogous transversely directed force also occurs in response to energy flow. Quantum mechanics treats the energy emanating from stars, galaxies, and other celestial objects as an observable. We require instead a theory of emission that is derived at the quantum level, treats energy as a flow rather than anservable, and exhibits effects due to transverse fields analogous to those of gravitomagnetics. The particle model of energy emission given by 4) will be helpful in the development of a model that includes transverse fields.

To compare the flow of gravitational energy with its electromagnetic counterpart we require a four-dimensional field interpretation of the creation process described by 4 ). This is possible by following Dirac's suggestion to formulate quantum mechanics in a space-time region in terms of a "vibrating medium" and use a Lagrangian density given by the fields and its first derivatives $L\left(\phi_{i}, \phi_{i, \mu}\right)[11]$. The idea begins from a classical vantage point. "We introduce at each point of space-time a Lagrangian density, which must be a function of the coordinates and their first derivatives with respect to $x, y, z$, and $t$, corresponding to the Lagrangian in particle theory being a function of coordinates and 
velocities. The integral of the Lagrangian density over any (four-dimensional) region of space-time must then be stationary for all small variations of the coordinates inside the region, provided the coordinates on the boundary remain invariant." We can adapt Dirac's line of reasoning to the particle model of 4) by describing fields over a four-dimensional region of space-time with respect to invariant field boundaries coincident with the electron shells. Consider an atomic oscillator immersed in a radiation field with an outer electron that occupies either of two allowable energy states. Emission initiates from the excited state $R_{2}=\left(x_{2}, y_{2}, z_{2}\right)$ at time $t_{2}$ and it finalizes at the ground state $R_{1}=\left(x_{1}, y_{1}, z_{1}\right)$ at time $t_{1}$. Each of the energy states $R_{2}$ and $R_{1}$ determines a locus of points where the fields vanish and they may therefore be used to define invariant field boundaries. The integral of the Lagrangian density over the region of space-time between the excited and ground states is stationary for continuous variations of the coordinates inside the region; however, when discontinuous changes in action occur it is evaluated by integrating the Lagrangian density four-dimensionally, which is not zero as in classical theory but $\mathrm{h}$.

$$
S\left[\phi_{i}(t)\right]=\int_{R_{2}}^{R_{1}} \int_{t_{2}}^{t_{1}} L\left(\phi_{i} \phi_{i, \mu}\right) d^{3} x d t=h
$$

Because it is formulated with a time variable that is symmetrical with space the absorption and emission of radiation is described as a continuous flow, rather than as an infinite series of diagonalized energy states that use time as an independent background through which the states evolve. The action $S\left[\phi_{i}(t)\right]$ describes a four-dimensional localization of field that varies due to gravitational acceleration between the events $t_{2}$ and $t_{1}$, and a kinematic time dilation of electromagnetic fields that is continuous. It is a functional, a function of the values of coordinates on the discrete boundaries of the space-time surfaces $\mathrm{R}_{2}$ and $R_{1}$ which are in turn functions of the continuous space-time variables of the fields within the surface. The boundary of the space-time region uniquely fixes the interior. The relativistic model of energy flow explains both the mobility of energy and its localization as a concentration of field, the photon.

Inspection of equation 4) reveals no compelling reason for energy to be absorbed in discrete packets. In other words, it is possible for the atom to act as an intermediary of quantization by erecting field boundaries at $R_{1}$ and $R_{2}$ thereby localizing superposed electromagnetic waves. Emission follows with an electron located at the space-like potential $\mathrm{R}_{2}$ at time $\mathrm{t}_{2}$ and ends in the ground state with exact potential $\mathrm{R}_{1}$ at time $\mathrm{t}_{1}$. It is meaningless to speak of the "position of an electron" when describing the emission and absorption of energy since there are an infinite number of possible departure and arrival points on a space-like locus of points. The equipotential space-like surfaces representing the locus of points of all possible electron positions in an excited state and a ground state are the electron shells. Ideally the surfaces would be spherically shaped, but they are unobservable in principle since the observation of microscopic surfaces demands simultaneous detections, which due to special relativity theory are not allowable. As noted, three degrees of freedom are necessary to locate an electron on the surfaces of the electron shells and three to describe its path through the intervening space, where any trajectory is possible so long as it is consistent with these restraints. The wave function is inadequate because it has only three degrees of freedom to describe both the electron shell and motion between the shells, and also because the wave function does not allow discrete and continuous time to appear in its equations simultaneously.

Clock function is easily interpreted using equation 6). The unbounded transverse field energy from a laser is absorbed by a lattice of atoms, localized within four-dimensional field boundaries, and then emitted to produce a single clock cycle. Solving for the action we obtain the same result, $E \tau=h$, as in section 3.1. Thus, when energy is absorbed by an atom it is localized between two space-like equipotential surfaces and is subsequently released as a photon. The emission equation 6) gives both the discrete and continuous aspects of time, an important difference with the equations of nonrelativistic quantum mechanics where the wave function is defined continuously in time. 


\subsection{Relativistic absorption of energy}

In the relativistic equations 4) and 6) the excitation energy is quantized and it appears as a localization of fields. It means that a physical separation exists between the matter and energy of an excited state. An electron does not receive, or absorb energy when it is excited, rather excitation causes field boundaries to be erected that localize energy within the atom and create a "bound" photon. In fact, the existence of locally autonomous photons within an atom has been demonstrated in experiments referred to as "the phenomena of slow or stopped light" [12]. The storage and retrieval of light are achieved for up to one minute in a rare earth element by converting light coherence in free space to atomic coherence in an excited state and back again. The atom establishes discrete field boundaries that localize energy, while the transverse fields contained within them vary independently and are continuous in time.

\section{Gravitational field energy}

\subsection{Field transformation}

In the previous paragraph we conceived of quantization as the absorption of electromagnetic field energy by a linear superposition of field potentials that occurs continuously over a time period equal to the wave period and transforms by a four-dimensional localization of fields into the photon. The transformation of fields can also be expressed classically according to Maxwell's equations, as the superposition of transverse radiation fields that begin in free space as linear potentials given by the vector potential alone,

\section{$B=\nabla \times A$}

and transform nonlinearly into a field source, the photon, with distinct field geometry. We are motivated to adopt the same method for describing the time evolution of gravitational fields in free space and their transformation into matter by hypothesizing that the laws of energy apply in an equivalent way. Fundamental to these arguments is the assumption that the laws of energy in nature such as energy conservation and equipartition, are universal. As is well known, the creation of a gravitational field source begins with a distribution of linear field potentials in free space which coalesce non-linearly to create a black hole at a discrete point in time. Existing attempts to unify the gravitational and electromagnetic fields use a particle model and seek to derive equations to determine the behavior of test particles in a combined field with respect to the conjugate variables position and momentum. Yet we know that real unification already exists at the particle level as embodied in the structure of electrons and other elementary particles. Rather than address the issue of unification from the vantage point of the particle we seek equations that use the conjugate variables energy and time, and seek to describe the time evolution of electromagnetic and gravitational field energy as flows. They are physically equivalent descriptions and may be used in place of particle properties to obtain an alternative view of the emission and absorption processes.

\subsection{General relativistic time}

We wish to describe gravitational field energy similarly to the way electromagnetic field energy is described, as the time evolution of fields that begin in free space as linear potentials. Time evolution has different meanings as it is used in quantum mechanics and general relativity theory. The time variable is given by the ticks of a clock in the former by using atomic clocks and the linearity of light as measurement standards, while the latter gives the proper time independently of clocks. Clocks are undefined in general relativity theory because Einstein believed that "measuring rods and clocks would have to be represented as solutions of the basic equations" [13]. Although he was unable to extend the equations to incorporate clocks or measuring rods we can see how differences between theory and practice originate by looking at the Einstein equation defining the local curvature of space-time.

$$
\mathrm{G}_{\mu \nu}=\kappa \mathrm{T}_{\mu \nu}
$$


Time and clocks are treated in a fundamentally different way in this equation. Time is continuous on the left side in the form of proper time, with clocks and measuring rods present as test bodies. If clocks, or matter of any type is present in significant amounts it must be placed on the right side due to contributions of mass and energy. Thus, continuous time exists in free space whether or not clocks are present, while clocks are functionally distinct because they are described by the discrete ticks of a clock. The equation lacks symmetry because there can be ideal clocks on either side, but real clocks are only allowable on the right.

\subsection{Universal properties of energy}

In classical theory we treat energy as a simple physical variable or property of matter and it is possible to use the same unit of measurement, joules, to measure all of its forms. Since only quantitative comparisons are possible in the measurement of joules the universal properties of energy are under appreciated or ignored. This is understandable in the case of classical interactions since systems are not precisely defined. Nevertheless, we see the same practice in quantum mechanics where energy is described as an observable in the Schrodinger time independent wave equation. Because each of the myriad forms of energy is conceived of separately with its own independent properties, we cannot compare them with each other. This is true despite the fact that universal properties of energy do exist. Energy conservation and energy equipartition, for example, have never been known to fail at any level of analysis whether microscopic or macroscopic. As noted in the study of atomic clock experiments it is possible to isolate a quantum system from the environment and describe it four-dimensionally as the transformation of electromagnetic field energy into matter. Gravitational systems in the form of galaxies are centers of energy emission and absorption naturally isolated from the environment and it should therefore be possible to compare the way they transform energy with that of a quantum system despite their vast physical differences. It is thereby postulated that the properties of energy, when described four-dimensionally with time, are independent of the material system which supports them.

\subsection{Transformation of gravitational field energy}

The four-dimensional evolution of gravitational fields may be demonstrated by constructing a model for a gravitating body that has slowly changing mass-energy density. We begin with a uniform distribution of hydrogen atoms, a simple form of clock, in free space distant from each other. Both proper time and clock time are determined in Minkowski spacetime and the gravitational field intensity of the system at infinity is found by simply summing particle masses. Now let the attractive force of their mutual gravitational field cause them to slowly coalesce into a spherical body such that particle momenta remain small. Due to the equivalence of mass and energy gravitational field intensity is determined by summing particle masses and binding energy, the energy required to remove particles to infinity. The period of clocks slows and Minkowski space-time is replaced by a Riemannian metric that is described by the Einstein tensor $G_{\mu v}$. An attempt to use spacetime curvature to describe material structure would be unacceptable because it would place the continuous time of curvature on a more fundamental basis than the ticks of a clock, a quantum mechanical phenomenon. A background space that is continuous cannot be used to describe discrete phenomena that take place within the background space.

Accumulating atoms will cause an increasing gravitational pressure that generates heat and the emission of black body radiation, early signs of star formation. Eventually the Coulomb repulsion of the atoms is overcome by gravitational attraction in the form of a pressure gradient, protons are attracted to each other due to the strong force, and fusion initiates spontaneously in the star's core. The left side of the Einstein equation 7) interprets the star formation process as a continuous change in metric from Minkowski space to a Riemannian manifold with constant proper time. On the other hand, on the right side there is a slowing of clocks with the accumulation of matter and a corresponding dilation 
of proper time. After matter has accumulated over a time period of several billion years proper time slows to zero on a spherical surface, the event horizon, and a black hole forms with discrete time $(\Delta t=0)$. We may refer to the event horizon therefore as a boundary condition with a space like surface and singular clock time.

\subsection{Gravitational emission equation}

In equation 6) we described the localization of electromagnetic field energy and photon creation four-dimensionally, using space-like field boundaries and discrete times. The localization of gravitational field energy is also characterized by the formation of a spacelike field boundary, the horizon, with discrete time. The similarity between the energy flow of photon creation and the energy flow of black hole formation can be extended to show a formal relationship. The Tully-Fisher relation correlates the luminosity and rotation velocity of galaxies [14]. If the luminosity of a complete revolution is calculated together with a galaxy's period of rotation $\tau$ then the Tully-Fisher relation may be compared to the quantization of electromagnetic fields, $\mathrm{E} \tau=\mathrm{h}$. The assumption that the laws of energy apply universally in nature allows an equivalent equation, $\mathrm{E} \tau=\mathrm{H}$, to be formulated for the transformation of gravitational field energy, where $\mathrm{H}$ is the gravitational equivalent of Planck's constant. Thus, an increased galactic emission energy, or energy flow will correspond to a higher rotational velocity and a lower period of rotation.

It is a short step from postulating that the Tully-Fisher correlation represents the solutions of an emission equation to obtaining the equation. The action integral $S\left[\Phi_{i}(t)\right]$ of a galaxy is a functional. That is, it is a function of the values of the space-like coordinates on an event horizon $\mathrm{R}$ which are in turn a function of the continuous space-time variables of the matter within the surface, where integration is performed over all space.

$$
S\left[\Phi_{i}\right]=\int_{R}^{\infty} \int_{t(0)}^{t(r)} L\left(\Phi_{i}, \Phi_{i, \mu}\right) d^{3} x d t=\mathrm{H}
$$

The existence of an emission equation governing galaxy energy flow suggests that galaxy structure is the manifestation of a field emanating from a supermassive black hole that consists of a radial component $\Phi_{\mathrm{i}}$ due to baryonic mass causing gravitational attraction and transverse components $\Phi_{\mathrm{i}, \mu}$ of as yet undetermined origin within the event horizon causing rotational acceleration. This hypothesis is supported by observational evidence that every galaxy has a black hole at its center and rotational acceleration is directly proportional to luminosity [15]. The forces are mapped on a flat background space in a reference system whose origin resides at the center of the black hole. The equations 6) and 8 ) both describe the flow of energy of a material system, atom and black hole, by including radial and transverse components. The fact that the emission equations are mathematically equivalent demonstrates a symmetry that is absent from theories that postulate the existence of unobserved forms of "dark" matter.

\section{Conclusion}

A fundamental difficulty that is encountered when attempting to unify quantum mechanics and general relativity theory is how to resolve their physically distinct concepts of space and time; that is, whether to adopt the discrete background space of quantum mechanics or the continuous, curved space-time of general relativity theory. We have shown in the previous arguments that both the Minkowski and Riemannian space-times are needed. Minkowski space describes atomic structure by allowing the equivalence principle to be applied to electron transitions in the manifestly covariant process of energy absorption and emission, while the atoms themselves reside in the curved Riemannian space-time geometry of general relativity theory. Both the flat space-time of atomic structure and the curved space-time that resides between particles are needed to compare the time evolution of electromagnetic and gravitational fields during transformations of energy. Paradoxes and misunderstandings arise when Minkowski space is used to interpret events that occur in the classically defined space-time of general relativity theory. 
The interdependent nature of the conjugate variables should now be clear. We use the position and momentum variables of test particles to map local configurations of the electric, magnetic, and gravitational fields. The energy and time variables, on the other hand, are used to describe nonlocal, globally determined coordinates both in space, as manifested by the space like surfaces of electron shells and the event horizons of black holes, and also in time by transformations of energy and the formation of galactic structure over time periods that potentially span the life of the universe. Descriptions by means of the conjugate variables energy and time supersede locally defined descriptions with respect to position and momentum which do not accurately reflect the time evolution of clock mechanisms or the continuous flow of electromagnetic and gravitational energy during absorption and emission processes.

\section{References}

[1] Hinkley, N., Sherman, J. A. et al. “An atomic clock with 10-18 instability" (2013).

ArXiv:1305.5869 69

[2] Takamoto, M., Ushijima, I., et al. "Test of general relativity by a pair of transportable optical lattice clocks" Nat Photonics (2020). DOI: $10.1038 / \mathrm{s} 41566-020-0619-8$

[3] Rothman, T.; Boughn, S. (2006). "Can Gravitons be Detected?". Found Phys 36 (12): arXiv:gr-qc/0601043. doi:10.1007/s10701-006-90819. S2CID 14008778.

[4] Dirac, P.A,M. "The quantum theory of the emission and absorption of radiation" Proc Roy Soc A 114, 243 (1927).

[5] Hafele, J. C., Keating, R. E., “Around-the-world atomic clocks” Science 177 (1972), p. 166.

[6] Marti, G. E., Hutson, R. B., “Imaging optical frequencies with $100 \mu \mathrm{Hz}$ precision and $1.1 \mu \mathrm{m}$ resolution”, PRL 120, (2018), p.103201. DOI: 10.1103/PhysRevLett.120.103201

[7] T. Bothwell et al. "Resolving the gravitational red shift within a millimeter atomic sample". arXiv:2109.12238.

[8] R. V. Pound, G. A. Rebka Jr., PRL 3 (9) 439 (1959) doi:10.1103/PhysRevLett.3.439

[9]Minkowski, H. "Das Relativitats Prinzip", Ann Phys 352 (15): 927-938.(1907) doi:10.1002/andp.19153521505.

[10] Williams, R.K. "Gravitomagnetic field and Penrose scattering processes". Annals of the New York Academy of Sciences 1045 (2005) pp. 232-245.

[11] Dirac, P.A.M., “The Lagrangian in quantum mechanics” Phys Zeit Sow 3 (1933), p.1.

[12] Heinze, G., Hubrich, C., and Halfmann, T., "Stopped light and image storage by electromagnetically induced transparency up to the regime of one minute", PRL 111, (2013), p. 033601. DOI: 10.1103/PhysRevLett.111.033601

[13] A. Einstein in P. A. Schilpp (ed.), Albert Einstein: Philosopher-Scientist, Vol. 7, (Open Court: Illinois, 1949), p. 59.

[14] Tully SR.B. \& Fisher, J.R. “A new method of determining distances to galaxies” Astron Astrophys 54 (1977), p. 661. 